\title{
The Vitali covering theorem in constructive mathematics
}

\author{
HANNES DIENER \\ ANTON HEDIN
}

\begin{abstract}
This paper investigates the Vitali Covering Theorem from various constructive angles. A Vitali Cover of a metric space is a cover such that for every point there exists an arbitrarily small element of the cover containing this point. The Vitali Covering Theorem now states, that for any Vitali Cover one can find a finite family of pairwise disjoint sets in the Vitali Cover that cover the entire space up to a set of a given non-zero measure. We will show, by means of a recursive counterexample, that there cannot be a fully constructive proof, but that adding a very weak semi-constructive principle suffices to give such a proof. Lastly, we will show that with an appropriate formalization in formal topology the non-constructive problems can be avoided completely.
\end{abstract}

2010 Mathematics Subject Classification 03F60, 28E99 (primary); 03F55, 06D22 (secondary)

Keywords: constructive mathematics, reverse mathematics, measure theory, Vitali's covering theorem, formal topology

\section{Introduction}

The Vitali Covering Theorem (VCT), from 1908 and due to Italian Giuseppe Vitali [18], is a result in the measure theory of Euclidean spaces. In its simplest form ${ }^{1}$ it can be stated as follows.

Theorem 1 Let $\mathcal{V}$ be a Vitali cover of a set $X \subseteq \mathbb{R}$ with finite outer measure. Then there is a sequence $\left(I_{n}\right)_{n \geq 1}$ of pairwise disjoint elements of $\mathcal{V}$ such that

$$
\mu\left(X \backslash \bigcup_{i \geq 1} I_{i}\right)=0
$$

\footnotetext{
${ }^{1}$ More general versions of VCT consider subsets of $\mathbb{R}^{n}$ and the Hausdorff measure rather than the Lebesgue one.
} 
Here a Vitali cover of a set $X \subseteq \mathbb{R}$ is a family of intervals $\mathcal{V}$ such that for every point $x \in X$ and $\varepsilon>0$, the cover $\mathcal{V}$ contains some interval $I$ with $x \in I$ and length $|I|<\varepsilon$.

One well known application of VCT is Lebesgue's Differentiation Theorem [12]: If $f$ is continuous and non-decreasing on $[a, b] \subseteq \mathbb{R}$, then $f^{\prime}(x)$ exists almost everywhere on $[a, b]$ (For a proof using VCT see [6, Ch. 4.4]).

Since VCT is about extracting a sub-family from a given cover with certain properties, it is superficially similar to the Heine-Borel Theorem. The key difference is that in VCT one is interested in extracting a disjoint family of sets. The usual classical proof (see e.g. [6, Ch. 4] for a proof due to Banach) is unacceptable from a constructive point of view. We will show that there cannot be a fully constructive proof by giving a recursive counterexample, i.e. we show that in Russian constructive mathematics (RUSS) one can construct a Vitali cover for which even a weakening of the above Theorem 1 fails.

In Simpson's program of (Classical) Reverse Mathematics [16] VCT has been shown to be equivalent to a very weak form of Kőnig's Lemma called Weak Weak König's Lemma (WWKL) [5]. We show that also constructively WWKL is equivalent to VCT. This way we not only classify the latter in the program of Constructive Reverse Mathematics, we also obtain a proof in Brouwer's intuitionism, since WWKL is easily seen to follow from the Fan Theorem.

However, seen from a different angle VCT is fully constructive. Using the theory of formal spaces [15] we are able to give a constructive proof of a formal version of the theorem. In formal topology a version of the Heine-Borel Theorem holds [7], and as one might expect this makes a proof of the VCT possible. We will also relate the formal versions of these two covering theorems to their point-wise counterparts via the notion of spatiality of formal topologies.

\section{Preliminaries}

\subsection{Constructive mathematics}

By constructive mathematics we mean Bishop-style constructive mathematics (BISH) [3]; that is mathematics with intuitionistic logic together with some appropriate settheoretic foundation, such as Aczel's constructive set theory CZF [1].

We will also talk about two other schools of constructive mathematics. Namely, the above-mentioned schools of Russian constructive mathematics and Brouwer's intuitionism (INT). In relation to BISH, these disciplines can be seen as extensions 
obtained by adding, in the first case a Church-Turing type principle, and in the second case a continuity principle and the principle of bar induction. For further details on BISH, RUSS and INT we refer to [4].

Following Bishop we make free use of the axiom of countable choice, especially in Section 4.

\subsection{Intervals and measure}

For simplicity and clarity's sake we will consider the following simpler restatement of Theorem 1, which avoids any reference to a measure. It seems feasible to extend the proofs in Sections 4 and 5 to a more general setting, but we believe that even though this could be done easily, it would not contribute to a better understanding of the Vitali covering theorem.

Theorem 2 Let $\mathcal{V}$ be a countable Vitali cover of $[a, b] \subseteq \mathbb{R}$. Then there exists a sequence $\left(I_{n}\right)_{n \geq 1}$ of pairwise disjoint intervals of $\mathcal{V}$ such that for each $\varepsilon>0$ there is $N \in \mathbb{N}$ such that

$$
\sum_{i=1}^{N}\left|I_{i}\right|>b-a-\varepsilon .
$$

For an interval $I \subseteq \mathbb{R}$ we denote by $\underline{I}$ and $\bar{I}$ its left and right endpoints respectively. Moreover we denote by $|I|$ the length of $I$, that is $|I|=\bar{I}-\underline{I}$. Furthermore, by setting $|\emptyset|=0$, one ensures that the real number

$$
\mu\left(I_{1} \cup \cdots \cup I_{n}\right)=\sum_{k=1}^{n}\left|I_{k}\right|-\sum_{k<j}^{n}\left|I_{k} \cap I_{j}\right|,
$$

exists for any finite collection of intervals $I_{1}, \ldots, I_{n} \subseteq \mathbb{R}$. Thus it will be clear what we mean by $\mu\left(J \backslash \bigcup_{i=1}^{n} I_{i}\right)$, for intervals $J, I_{1}, \ldots, I_{n}$. Unless otherwise stated all intervals we consider will be non-trivial, that is intervals with $\underline{I}<\bar{I}$.

Even though measure theory can be satisfactorily treated constructively [3, Chapter 6] we can, with the chosen formalizations, completely avoid it.

The following combinatorial result is commonly referred to as the "Baby Vitali Lemma" and is a key intermediate step in the proof of VCT common to all approaches.

Lemma 3 Given finitely many intervals $I_{1}, \ldots, I_{n} \subseteq \mathbb{R}$, we can find a pairwise disjoint subsequence $I_{k_{1}}, \ldots, I_{k_{m}}$ such that

$$
\mu\left(I_{k_{1}} \cup \ldots \cup I_{k_{m}}\right) \geq \frac{1}{4} \mu\left(I_{1} \cup \ldots \cup I_{n}\right) .
$$


Proof Choose intervals $J_{1}, \ldots, J_{n}$ with rational endpoints such that $I_{j} \subseteq J_{j}$ and

$$
\left|J_{j}\right| \leq \frac{4}{3}\left|I_{j}\right|
$$

for all $1 \leq j \leq n$. Choose $k_{1}$ such that $\left|J_{k_{1}}\right| \geq\left|J_{j}\right|$ for every $1 \leq j \leq n$. Suppose $k_{1}, \ldots, k_{l}$ have been chosen, then choose $k_{l+1}$ such that $\left|J_{k_{l+1}}\right| \geq\left|J_{j}\right|$ for each $1 \leq j \leq n$ with $J_{j}$ disjoint from $J_{k_{1}}, \ldots, J_{k_{l}}$. This process eventually stops and we get a subsequence $J_{k_{1}}, \ldots, J_{k_{m}}$ of pairwise disjoint intervals. Moreover, for any $1 \leq j \leq n$ there is $1 \leq l \leq m$ such that $J_{j} \cap J_{k_{l}}$ is inhabited and $\left|J_{j}\right| \leq\left|J_{k_{l}}\right|$.

Now we widen the chosen intervals by letting

$$
J_{k_{j}}^{\prime}=\left(2 \underline{J_{k_{j}}}-\overline{J_{k_{j}}}, 2 \overline{J_{k_{j}}}-\underline{J_{k_{j}}}\right)
$$

so that $\left|J_{k_{j}}^{\prime}\right|=3\left|J_{k_{j}}\right|$ and $J_{k_{j}}^{\prime}$ and $J_{k_{j}}$ have the same midpoint for each $1 \leq j \leq m$. Then, for any $1 \leq j \leq n$ there is $1 \leq l \leq m$ such that $J_{j} \subseteq J_{k_{l}}^{\prime}$ and hence

$$
\mu\left(J_{1} \cup \cdots \cup J_{n}\right) \leq \mu\left(J_{k_{1}}^{\prime} \cup \cdots \cup J_{k_{m}}^{\prime}\right)=3 \mu\left(J_{k_{1}} \cup \cdots \cup J_{k_{m}}\right) .
$$

The sequence $I_{k_{1}}, \ldots, I_{k_{m}}$ then satisfies

$$
\begin{aligned}
\mu\left(I_{k_{1}} \cup \cdots \cup I_{k_{m}}\right) & \geq \frac{3}{4} \mu\left(J_{k_{1}} \cup \cdots \cup J_{k_{m}}\right) \\
& \geq \frac{1}{4} \mu\left(J_{1} \cup \cdots \cup J_{n}\right) \\
& \geq \frac{1}{4} \mu\left(I_{1} \cup \cdots \cup I_{n}\right) .
\end{aligned}
$$

Remark 4 In case the intervals $I_{1}, \ldots, I_{n}$ all have rational endpoints the constant in the Baby Vitali Lemma can be sharpened to $\frac{1}{3}$, which is the constant appearing in the classical statement of the Lemma.

The proofs of VCT in Sections 4 and 5 both reduce to proving the following property for a Vitali cover $\mathcal{V}$ and some $c \in(0,1)$.

$\left(V_{c}\right)$ For any interval $[a, b]$ for which $\mathcal{V}$ is a Vitali cover, there exist finitely many pairwise disjoint intervals $I_{1}, \ldots, I_{n}$ in $\mathcal{V}$ such that $I_{i} \subseteq(a, b)$ for each $i=1, \ldots, n$ and

$$
\mu\left([a, b] \backslash \bigcup_{i=1}^{n} I_{i}\right)<c(b-a) .
$$


We may assume that every interval $I_{k}$ in $V_{c}$ is non-trivial: for any of them we can decide whether $\left|I_{k}\right|>0$ or

$$
\left|I_{k}\right|<c(b-a)-\mu\left([a, b] \backslash \bigcup_{i=1}^{n} I_{i}\right) ;
$$

in the second case we can simply drop it without changing equation (1). If $\mathcal{V}$ has this property $V_{c}$ then the proof of Theorem 2 is straightforward.

Proof of Theorem 2 Suppose the covering $\mathcal{V}$ has the property $\left(V_{c}\right)$. Choose $\mu>0$ such that $c+\mu<1$. By $\left(V_{c}\right)$ we get a finite subset $\mathcal{J}_{0}=\left\{I_{1}, \ldots, I_{n}\right\} \subseteq \mathcal{V}$ of pairwise disjoint intervals such that $\sum_{i=1}^{n}\left|I_{i}\right|>(1-c)(b-a)$ and $I_{i} \subseteq(a, b)$ for each $1 \leqslant i \leqslant n$. Following the remark above we can assume that the intervals are non-trivial, so we can decide for $i \neq j$ whether $\underline{I}_{i}>\underline{I_{j}}$ or $\underline{I_{i}}<\overline{I_{j}}$. In the second case, by the disjointness of the intervals, $\overline{I_{i}} \leqslant \underline{I_{j}}$. Thus without loss of generality we may assume that $\overline{I_{i}} \leqslant \underline{I_{i+1}}$ for all $1 \leqslant i<n$.

Now set $J_{0}=\left[a, \underline{I}_{1}\right], J_{k}=\left[\bar{I}_{k}, I_{k+1}\right]$ for $k=1, \ldots, n-1$, and $J_{n}=\left[\bar{I}_{n}, b\right]$. Construct a decidable $T \subseteq\{0, \ldots, n\}$ such that $k \in T$ implies that

$$
\left|I_{k}\right|<\delta=\mu c(b-a) /(n+1),
$$

and $k \notin T$ implies that $I_{k}$ is non-trivial.

As $\mathcal{V}$ is a Vitali cover of any subinterval of $[a, b]$, we can apply the property $\left(V_{c}\right)$ again to every $J_{i}$ where $i \notin T$ and get a finite subset $\mathcal{I}_{i} \subseteq \mathcal{V}$ of pairwise disjoint intervals such that

$$
\mu\left(J_{i} \backslash \bigcup \mathcal{I}_{i}\right)<c\left|J_{i}\right|
$$

In case that $i \in T$, that is when $J_{i}$ is very small, we set $\mathcal{I}_{i}=\emptyset$. For every $k$ the elements of $\mathcal{I}_{k}$ are contained in the interior of $J_{k}$. Hence, if we let $\mathcal{J}_{1}=\mathcal{J}_{0} \cup \bigcup_{i=0}^{n} \mathcal{I}_{i}$, it is straightforward that the elements of $\mathcal{J}_{1}$ are pairwise disjoint and that

$$
\begin{aligned}
\mu\left([a, b] \backslash \bigcup \mathcal{J}_{1}\right) & <\sum_{i=0}^{n} \mu\left(I_{i} \backslash \bigcup \mathcal{J}_{1}\right) \\
& <\sum_{i \notin T} \mu\left(I_{i} \backslash \bigcup \mathcal{J}_{1}\right)+\sum_{i \in T} \mu\left(I_{i}\right) \\
& <c^{2}(b-a)+(n+1) \delta \\
& =(c+\mu) c(b-a)
\end{aligned}
$$


Repeating this process $m$ times we end up with a finite subset $\mathcal{J}_{m} \subseteq \mathcal{V}$ of pairwise disjoint intervals such that

$$
\mu\left([a, b] \backslash \bigcup \mathcal{J}_{m}\right)<(c+\mu)^{m-1} c(b-a),
$$

and $\mathcal{J}_{m-1} \subseteq \mathcal{J}_{m}$. Enumerating $\bigcup_{n \in \mathbb{N}} \mathcal{J}_{n}$ yields a sequence $\left(I_{n}\right)_{n \geqslant 1}$ with the desired property: Let $\varepsilon>0$ and choose $N \in \mathbb{N}$ such that $(c+\mu)^{N-1} c(b-a)<\varepsilon$. Furthermore let $M$ be large enough, such that $\mathcal{J}_{N} \subseteq\left\{I_{1}, I_{2}, \ldots, I_{M}\right\}$. Then

$$
\mu\left([a, b] \backslash \bigcup_{i=1}^{M} \mathcal{I}_{i}\right)<(c+\mu)^{N-1} c(b-a)<\varepsilon ;
$$

or equivalently, since the intervals are pairwise disjoint,

$$
\sum_{i=1}^{M}\left|I_{i}\right|>b-a-\varepsilon \text {. }
$$

\subsection{Formal Spaces}

Formal topology is a constructive version of the point-free approach to topology developed under the name of locale theory [11]. We will recall some basic notions necessary for later sections. For further details see [15].

Let $(X, \leqslant)$ be a pre-ordered set, i.e. $\leqslant$ is reflexive and transitive. For any subsets $U, V \subseteq X$ we let $\downarrow U \stackrel{\text { def }}{=}\{x \in X:(\exists u \in U) x \leqslant u\}$ and $U \wedge V \stackrel{\text { def }}{=} \downarrow U \cap \downarrow V$. We will moreover use the notation $U \zeta V \stackrel{\text { def }}{\Longleftrightarrow}(\exists u \in X) u \in U \cap V$.

Definition 5 A Formal Topology $\mathcal{X}=(X, \leqslant, \triangleleft)$ consists of a pre-ordered set $(X, \leqslant)$ together with a relation $\triangleleft$ between elements of $X$ and subsets of $X$ satisfying the following conditions

(Ref) $a \in U \Longrightarrow a \triangleleft U$,

(Tra) $a \triangleleft U \& U \triangleleft V \Longrightarrow a \triangleleft V$,

(Ext) $a \leqslant b \Longrightarrow a \triangleleft\{b\}$,

(Loc) $a \triangleleft U \& a \triangleleft V \Longrightarrow a \triangleleft U \wedge V$.

Here $U \triangleleft V \stackrel{\text { def }}{\Longleftrightarrow}(\forall a \in U) a \triangleleft V$. We often write $a \triangleleft b$ instead of $a \triangleleft\{b\}$ for $a, b \in X$. 
A formal point of $\mathcal{X}=(X, \leqslant, \triangleleft)$ is a subset $\alpha \subseteq X$ satisfying

(P1) $(\exists x \in X) x \in \alpha$,

(P2) $a, b \in \alpha \Longleftrightarrow\{a\} \wedge\{b\} \gamma \alpha$.

(P3) $a \in \alpha \& a \triangleleft U \Longrightarrow \alpha \gamma U$.

We denote by $\operatorname{Pt}(\mathcal{X})$ the class of points of $\mathcal{X}$ and call it the formal space of $\mathcal{X}$. For $U \subseteq X$ we let $\operatorname{ext}[U] \stackrel{\text { def }}{=}\{\alpha \in \operatorname{Pt}(\mathcal{X}): \alpha \chi U\}$-the class of points containing some element of $U$. The spatial topology on $\operatorname{Pt}(\mathcal{X})$ is the topology $\tau_{\mathcal{X}}$ generated by the base $\operatorname{ext}[a], a \in X$.

We recall a well known construction of the real numbers as a formal space (see for example [11] and [13]). The formal topology of formal reals is the structure $\mathcal{R}=(R, \leqslant, \triangleleft)$, with $R \subseteq \mathbb{Q} \times \mathbb{Q}$ the set of all pairs $(p, q)$ where $p<q$. The pre-order is given by inclusion, i.e. $(p, q) \leqslant(r, s)$ if and only if $r \leq p$ and $q \leq s$. We also have the obvious strict version $<$. The cover is defined by

$$
(p, q) \triangleleft U \stackrel{\text { def }}{\Longleftrightarrow}\left[\forall\left(p^{\prime}, q^{\prime}\right)<(p, q)\right]\left(p^{\prime}, q^{\prime}\right) \triangleleft_{f} U,
$$

where $\triangleleft_{f}$ is inductively defined by the rules

(R1) $(p, q) \in U \Longrightarrow(p, q) \triangleleft_{f} U$,

(R2) $(p, s) \triangleleft_{f} U,(r, q) \triangleleft_{f} U, p \leq r<s \leq q \Longrightarrow(p, q) \triangleleft_{f} U$,

(R3) $(r, s) \triangleleft_{f} U,(p, q) \leqslant(r, s) \Longrightarrow(p, q) \triangleleft_{f} U$.

When two pairs $(p, q),(r, s)$ are consistent, i.e. $r<q$ and $p<s$, their meet exists in $R$ and is given by

$$
(p, q) \wedge(r, s)=(\max (p, r), \min (q, s)) .
$$

We will say that two pairs $(p, q)$ and $(r, s)$ in $R$ are disjoint when $s \leq p$ or $q \leq r$. For $U \subseteq R$ we denote by $\operatorname{Fin}(U)$ the set of finitely enumerable subsets ${ }^{2}$ of $U$.

Lemma 6 The following properties hold of the covers $\triangleleft_{f}$ and $\triangleleft$ :

(1) If $(p, q) \triangleleft_{f} U$ then $(p, q) \triangleleft U$,

(2) If $(p, q) \triangleleft_{f} U$, then there is $U_{0} \in \operatorname{Fin}(U)$ such that $(p, q) \triangleleft_{f} U_{0}$,

\footnotetext{
${ }^{2}$ In fact, as the elements of $R$ are pairs of rational numbers, $\operatorname{Fin}(U)$ is the same as the set of all finite subsets of $U$.
} 
(3) If $U$ is finite then $(p, q) \triangleleft_{f} U$ if and only if $\operatorname{ext}[(p, q)] \subseteq \operatorname{ext}[U]$,

(4) If $(p, q) \triangleleft U$ and $U$ is finite, then $(p, q) \triangleleft_{f} U$.

Proof For (1) suppose $(r, s)<(p, q) \triangleleft_{f} U$, then $(r, s) \leqslant(p, q)$ and $(r, s) \triangleleft_{f} U$ follows since $\triangleleft_{f}$ is a cover.

Item (2) is proved by a straightforward induction on the finitary cover $(p, q) \triangleleft_{f} U$.

For (3) the left to right direction is immediate. Conversely, suppose ext $[(p, q)] \subseteq \operatorname{ext}[U]$ for $U$ finite. Since $U$ is finite and covers $(p, q)$ (as intervals) we may choose $\left(p_{i}, q_{i}\right) \in U$, $i=0, \ldots, n$, such that $p_{0} \leq p<q_{0}, p_{n}<q \leq q_{n}$ and $p_{i+1}<q_{i}$ for $i=0, \ldots, n-1$. It is then clear that $\left(p_{0}, q_{n}\right) \triangleleft_{f} U$ (by repeated use of (R2)) from which $(p, q) \triangleleft_{f} U$ follows by (Ext).

The proof of item (4) is much more involved, and we refer to [7] for the details.

The points of $\mathcal{R}$, which we call formal reals, can be characterized as follows.

Lemma $7 \quad A$ subset $\alpha$ of $R$ is a formal real if it satisfies

(P1) $\alpha$ is inhabited,

(P2) $(p, q),(r, s) \in \alpha$ if and only if $(p, q) \wedge(r, s) \in \alpha$,

(P3) $(p, q) \in \alpha$ and $p \leq r<s \leq q$ implies $(p, s) \in \alpha$ or $(r, q) \in \alpha$,

(P4) $(p, q) \in \alpha$ implies there is $(r, s) \in \alpha$ with $(r, s)<(p, q)$.

The collection of formal reals, denoted $\operatorname{Pt}(\mathcal{R})$, is precisely the set of Dedekind reals and with countable choice we can show that $\operatorname{Pt}(\mathcal{R})$ is homeomorphic to the Cauchy reals à la Bishop [13]. The rational numbers are embedded via

$$
r \mapsto\{(p, q) \in R: p<r<q\},
$$

and we will without further mention use letters $p, q, r, s, \ldots$ for denoting rationals both as basic numbers and formal reals. The arithmetic order on $\operatorname{Pt}(\mathcal{R})$ is given by

$$
\alpha<\beta \stackrel{\text { def }}{\Longleftrightarrow}(\exists(p, q) \in \alpha)(\exists(r, s) \in \beta) q<r,
$$

and we define $\alpha \leq \beta \stackrel{\text { def }}{\Longleftrightarrow} \neg(\beta<\alpha)$. Thus, when we write $r<\alpha$ we really mean $\{(p, q) \in R: p<r<q\}<\alpha$ which by definition means that there is $(p, q) \in r$ and $(u, v) \in \alpha$ with $q<u$. The next property of formal reals will be useful in the following sections. 
Lemma 8 If $\alpha$ is a formal real, then for any $k>0$ there exists $(p, q) \in \alpha$ with $q-p<k$.

Proof Let $\alpha \in \operatorname{Pt}(\mathcal{R})$ and $k>0$. Let $(r, s) \in \alpha$, then $\left(r, \frac{r+2 s}{3}\right) \in \alpha$ or $\left(\frac{2 r+s}{3}, s\right) \in \alpha$. Then let $n$ be large enough that $\left(\frac{2}{3}\right)^{n}(s-r)<k$ and we see that repeating the above trisection $n$ times will give us an element $(p, q) \in \alpha$ with $q-p<k$.

\section{A recursive counterexample}

Theorem 9 In RUSS there exists a Vitali cover for which Theorem 2 fails to hold.

Before we construct this counterexample, we remind the reader of the following recursive obscurity. A sequence of non-degenerate intervals $\left(I_{n}\right)_{n \geq 1}$ is called an $\alpha$-singular covering of $[0,1]$ if

(1) For all $m \neq n$ either $I_{n} \cap I_{m}=\emptyset, \underline{I_{n}}=\overline{I_{m}}$ or $\underline{I_{m}}=\overline{I_{n}}$.

(2) For all $x \in[0,1]$ there exists $m, n$ with $\underline{I_{n}}=\overline{I_{m}}$ and $\underline{I_{m}}<x<\overline{I_{n}}$.

(3) For all $n \in \mathbb{N}$

$$
\sum_{i=1}^{n}\left|I_{i}\right|<\alpha .
$$

It is a theorem in RUSS that for any $0<\alpha$ there exists an $\alpha$-singular covering of $[0,1]$, see for example [2]. We are now in the position to give a proof of Theorem 9.

Proof Let $\left(J_{n}\right)_{n \geq 1}$ be a $\frac{1}{6}$-singular covering. We need to extend these intervals slightly, so that their union covers the unit interval. To this end set $I_{n}=\left(2 \underline{J_{n}}-\overline{J_{n}}, 2 \overline{J_{n}}-\underline{J_{n}}\right)$. Then

- $[0,1] \subseteq \bigcup_{i=1}^{\infty} I_{i}$ and

- $\sum_{i=1}^{n}\left|I_{i}\right|=3 \sum_{i=1}^{n}\left|J_{i}\right| \leq \frac{1}{2}$, for all $n \in \mathbb{N}$.

For each $n \in \mathbb{N}$ let $\left(I_{n}^{k}\right)_{k \geq 1}$ be an enumeration of all intervals with rational endpoints contained in $I_{n}$. Then the family of intervals $\left(I_{n}^{k}\right)_{k, n}$ is easily seen to be a Vitali cover. Now assume Theorem 1 holds; that is assume that there exist pairwise disjoint $I_{n_{1}}^{k_{1}}, \ldots, I_{n_{m}}^{k_{m}}$ such that

$$
\mu\left([0,1] \backslash \bigcup_{i=1}^{m} I_{n_{i}}^{k_{i}}\right)<\frac{1}{2} .
$$


Now

$$
\begin{aligned}
\frac{1}{2}>\mu\left([0,1] \backslash \bigcup_{i=1}^{m} I_{n_{i}}^{k_{i}}\right) & =1-\mu\left(\bigcup_{i=1}^{m} I_{n_{i}}^{k_{i}}\right) \\
& \geq 1-\mu\left(\bigcup_{i=1}^{m} I_{n_{i}}\right) \\
& \geq 1-\sum_{i=1}^{m} \mu\left(I_{n_{i}}\right) \\
& \geq \frac{1}{2} ;
\end{aligned}
$$

a contradiction.

We would like to mention that it can be shown that the Vitali cover constructed above is even totally bounded under the Hausdorff metric, thus limiting the possibility of a purely constructive version of VCT even under additional assumptions.

\section{An intuitionistic proof}

The recursive counterexample above relies on the existence of singular covers. In Brouwer's intuitionism the existence of such objects is ruled out by the Fan theorem-to be precise the Fan theorem for detachable bars suffices to ensure that any countable covering of $[0,1]$ by open intervals has a finite subcover [8]. As it turns out an even weaker principle suffices to prove Vitali's covering theorem. Researchers working on S. Simpson's program of classical reverse mathematics have proven that in their system Vitali's covering theorem is equivalent to a "weak weak" version of König's Lemma [5]. Without a change it is readily accessible as a principle in constructive reverse mathematics:

WWKL. If $B \subseteq 2^{*}$ is a decidable bar that is closed under extensions, then

$$
\lim _{n \rightarrow \infty} \frac{|\{u \notin B:|u|=n\}|}{2^{n}}=0 .
$$

We remind the reader that a bar $B$ is a subset of the set of all finite binary sequences $2^{*}$, such that for any infinite binary sequence $\alpha \in 2^{\mathbb{N}}$ there is a natural number $N$ such that 
its initial segment $\bar{\alpha} N$ of the first $N$ terms is in $B$. Also, $B$ is closed under extensions, if for all $u, w \in 2^{*}$ we have that

$$
u \in B \Longrightarrow u * w \in B
$$

where $u * w$ is the concatenation of $u$ followed by $w$. The principle WWKL has already been investigated in constructive reverse mathematics [14]. Here we are going to prove the following equivalence, which also holds in Simpson's program.

Proposition 10 The following statements are equivalent in BISH

(a) WWKL

(b) If $I_{n}$ is a cover of $[0,1]$ with open intervals, then for all $\epsilon>0$ there exists $N$ such that

$$
\mu\left(\bigcup_{n=1}^{N} I_{n}\right)>1-\epsilon
$$

Proof Let us assume that WWKL holds, let $\left(I_{n}\right)_{n \geq 1}$ be an open cover of [0,1], and let $\varepsilon>0$ be arbitrary. Write each $I_{n}$ as the union of countably many open intervals with rational endpoints $I_{n, m}$. Notice that these intervals still cover $[0,1]$.

For every $u \in 2^{*}$ we will define an interval $J_{u}$ iteratively the following way: let $J_{0}$ be the interval $\left[0, \frac{1}{2}\right]$ and $J_{1}=\left[\frac{1}{2}, 1\right]$, and let $J_{u * 0}$ be the left half and $J_{u * 1}$ be the right half of $J_{u}$. Since we are dealing with intervals with rational endpoints the predicate $P(u)$, defined by

$$
P(u) \Longleftrightarrow J_{u} \subset \bigcup_{n, m \leq|u|} I_{n, m},
$$

is decidable. We claim that

$$
B=\left\{u \in 2^{*}: P(u)\right\}
$$

is a decidable bar. For let $\alpha \in 2^{\mathbb{N}}$ be arbitrary and consider its image $x=F(\alpha)$ under the canonical embedding $F$ of Cantor space into the unit interval. Since $\left(I_{n, m}\right)_{n, m \geq 1}$ is a cover there exist $n_{0}, m_{0} \in \mathbb{N}$ with $x \in I_{n_{0}, m_{0}}$. Since $I_{n_{0}, m_{0}}$ is open there exists $M \geq n_{0}, m_{0}$ such that $\left(x-2^{-M+1}, x+2^{-M+1}\right) \subseteq I_{n_{0}, m_{0}}$; but then also

$$
J_{\bar{\alpha} M} \subseteq\left[x-2^{-M}, x+2^{-M}\right] \subseteq I_{n_{0}, m_{0}},
$$

and hence $P(\bar{\alpha} M)$, which means that $\bar{\alpha} M \in B$. This shows that $B$ is a decidable bar. The set $B$ is also closed under extensions, since for any $u, w \in 2^{*}$ with $u \in B$ we have

$$
J_{u * w} \subset J_{u} \subset \bigcup_{n, m \leq|u|} I_{n, m} \subset \bigcup_{n, m \leq|u * w|} I_{n, m} .
$$


Hence, by WWKL, there exists $N$ with

$$
\frac{|\{u \notin B:|u|=N\}|}{2^{N}}<\varepsilon,
$$

which implies that

$$
\mu\left(\bigcup_{\substack{u:|u|=N \\ u \notin B}} J_{u}\right)<\varepsilon
$$

So

$$
\begin{aligned}
& 1-\mu\left(\bigcup_{n=1}^{N} I_{n}\right) \leq \mu\left(\bigcup_{u:|u|=N} J_{u}\right)-\mu\left(\bigcup_{n=1}^{N} I_{n}\right) \\
& \leq \mu\left(\bigcup_{u:|u|=N} J_{u}\right)-\mu\left(\bigcup_{n, m=1}^{N} I_{n, m}\right) \\
& \leq \mu\left(\bigcup_{u:|u|=N} J_{u} \backslash \bigcup_{n, m=1}^{N} I_{n, m}\right) \\
& =\mu\left(\bigcup_{\substack{u:|u|=N \\
u \notin B}} J_{u} \backslash \bigcup_{n, m=1}^{N} I_{n, m}\right) \\
& +\mu\left(\bigcup_{\substack{u:|u|=N \\
u \in B}} J_{u} \backslash \bigcup_{n, m=1}^{N} I_{n, m}\right) \\
& \leq \mu\left(\bigcup_{\substack{u:|u|=N \\
u \notin B}} J_{u}\right)+\mu\left(\bigcup_{\substack{u:|u|=N \\
u \in B}} J_{u} \backslash \bigcup_{n, m=1}^{N} I_{n, m}\right) \\
& \leq \mu\left(\bigcup_{\substack{u:|u|=N \\
u \notin B}} J_{u}\right)+0 \\
& <\varepsilon \text {. }
\end{aligned}
$$

This proves that $(a) \Longrightarrow(b)$. 
In order to prove the reverse implication, assume that $(b)$ holds and let $B$ be a decidable bar which is closed under extensions. Moreover, let $\varepsilon>0$ be arbitrary. Now, iteratively, define intervals $\left(I_{u}\right)_{u \in 2^{*}}$ the following way: let $J_{u}$ be defined as above and $I_{u}$ be the open interval

$$
I_{u}=\left(\underline{J_{u}}-\frac{\varepsilon}{2^{2|u|+2}}, \overline{J_{u}}+\frac{\varepsilon}{2^{2|u|+2}}\right) .
$$

Since $B$ is decidable, we can enumerate a subset $\left(u_{n}\right)_{n \geq 1}$ of $B$ such that $\left\{u_{n}: n \geq 1\right\}$ is a bar and, for every $n \in \mathbb{N}$, either

- $\left\{u_{1}, \ldots, u_{n}\right\}$ is already a bar, or

- $\left\{u_{1}, \ldots, u_{n}\right\}$ are pairwise incomparable, i.e. no $u_{i}$ is a prefix of another.

Moreover, we may assume that $\left|u_{k}\right| \leq\left|u_{k+1}\right|$. Since $\left(u_{n}\right)_{n \geq 1}$ is a bar, the sequence $\left(I_{n}\right)_{n \geq 1}$, where $I_{n}=I_{u_{n}}$ for each $n \geq 1$, is a cover of [0,1]. Thus, applying $(b)$ we get $N \in \mathbb{N}$ such that

$$
\mu\left(\bigcup_{n=1}^{N} I_{n}\right)>1-\varepsilon
$$

Let $K=\left|u_{N}\right|$. Now, either $\left\{u_{1}, \ldots, u_{N}\right\}$ is already a bar, or consists of pairwise incomparable sequences. If the former holds we have

$$
\frac{|\{u \notin B:|u|=m\}|}{2^{m}}=0,
$$

for any $m \geq K$. If the latter holds we have, with $J_{n}=J_{u_{n}}$, that

$$
\mu\left(\bigcup_{n=1}^{N} I_{n}\right) \leq \sum_{n=1}^{N}\left|I_{n}\right|=\sum_{n=1}^{N}\left(\left|J_{n}\right|+\frac{\varepsilon}{2^{2\left|u_{n}\right|+1}}\right) \leq \sum_{n=1}^{N}\left|J_{n}\right|+\varepsilon
$$

and hence $\sum_{n=1}^{N}\left|J_{n}\right| \geq 1-2 \varepsilon$. Since $\left\{u_{1}, \ldots, u_{N}\right\}$ are pairwise incomparable we then have

$$
1-2 \varepsilon<\sum_{n=1}^{N}\left|J_{n}\right| \leq \sum_{\substack{u:|u|=K \\ u \in B}}\left|J_{u}\right|
$$

from which it follows that

$$
\frac{|\{u \notin B:|u|=K\}|}{2^{K}}<2 \varepsilon .
$$


Even though the proof above is very similar to the one given in [5] and [20], there are differences. Working with classical logic the law of excluded middle is available and used in Simpson's system. On the other hand the proof above uses countable choice, which is avoided in Simpson's framework. This is also a good time to point out that in [5] it is implicitly shown that-working in classical reverse mathematics-Vitali's covering theorem is equivalent to WWKL. Even though this indicates, that Vitali's covering theorem is not provable in RUSS, the construction above gives an explicit counterexample to that theorem.

Corollary 11 Assume WWKL holds. If $[a, b]$ is an interval and $\mathcal{V}$ a countable Vitali cover of $[a, b]$, then there exist finitely many, pairwise disjoint intervals $I_{1}, \ldots, I_{n}$ in $\mathcal{V}$ such that

$$
\sum_{k=1}^{n}\left|I_{k}\right|>\frac{1}{5}|a-b|,
$$

and $I_{k} \subseteq(a, b)$ for all $1 \leq k \leq n$.

Proof Follows from Lemma 3 and Proposition 10.

Thus, under the assumption that WWKL holds, we see that any countable Vitali cover of an interval $[a, b]$ has the property $\left(V_{\frac{4}{5}}\right)$, and thus the main theorem follows easily as noted in the preliminaries.

Theorem 12 (Intuitionistic Vitali Covering Theorem) Assume that WWKL holds. Let $\epsilon>0$ be arbitrary. If $\mathcal{V}$ is a countable Vitali cover of $[a, b]$, then there exists a finite set $\left\{I_{1}, \ldots, I_{m}\right\}$ of pairwise disjoint intervals of $\mathcal{V}$ such that

$$
\mu\left([a, b] \backslash \bigcup_{i=1}^{m} I_{i}\right)<\epsilon .
$$

It is worth pointing out that it is easy to see that VCT implies Part (b) of Proposition 10. Hence VCT is actually equivalent to WWKL.

\section{A point-free version}

The proof of the VCT for the formal reals relies on the Heine-Borel theorem. We therefore start by recalling some details of its proof [7] and discuss the relation to its failure in the point-wise case. 


\subsection{The Heine-Borel covering Theorem for $\mathcal{R}$}

By the Heine-Borel covering property for real numbers we mean the following.

HB. If $\left(I_{j}\right)_{j \in J}$ is a collection of open intervals covering $[0,1]$, then there is a finite subset $F \subseteq J$ such that $[0,1] \subseteq \bigcup_{j \in F} I_{j}$.

It is well known that $\mathbf{H B}$ fails in BISH because of the existence of recursive counterexamples in RUSS $^{3}$. On the other hand it has been shown that a version of $\mathbf{H B}$ holds for the formal reals [7]. This is no contradiction since on the formal side we restrict attention to covers $\left(I_{j}\right)_{j \in J}$ of open intervals (with rational endpoints) satisfying the relation $[0,1] \triangleleft\left\{I_{j}: j \in J\right\}$. We recall some of the details to make the last statement a bit more precise.

Given formal reals $\alpha \leq \beta$, we denote by $[\alpha, \beta]=\left(R, \leqslant, \triangleleft_{[\alpha, \beta]}\right)$ the closed sub-formal topology $\mathcal{R} \backslash \mathcal{C}[\alpha, \beta]$, where

$$
\mathcal{C}[\alpha, \beta]=\{(p, q) \in R: q<\alpha \vee \beta<p\} .
$$

By this we mean that the cover relation is given by

$$
(p, q) \triangleleft_{[\alpha, \beta]} U \stackrel{\text { def }}{\Longleftrightarrow}(p, q) \triangleleft U \cup \mathcal{C}[\alpha, \beta] .
$$

It is clear that $\triangleleft \subseteq \triangleleft_{[\alpha, \beta]}$. The formal space $\operatorname{Pt}([\alpha, \beta])$ is precisely the set of formal reals $\gamma$ satisfying $\alpha \leq \gamma \leq \beta$. Using the shorthand [ $\alpha, \beta] \triangleleft_{[\alpha, \beta]} U$ for $(\forall(p, q) \in R)(p, q) \triangleleft_{[\alpha, \beta]} U$, the formal Heine-Borel Theorem then states.

Theorem 13 If $[\alpha, \beta] \triangleleft_{[\alpha, \beta]} U$, then there exists a finite subset $U_{0} \subseteq U$ such that $[\alpha, \beta] \triangleleft_{[\alpha, \beta]} U_{0}$.

The following Lemma is crucial in the proof of the Theorem.

Lemma $14[\alpha, \beta] \triangleleft_{[\alpha, \beta]} U$ if and only if there exists $(r, s) \in R$ such that $r<\alpha \leq \beta<s$ and $(r, s) \triangleleft_{f} U \cup \mathcal{C}[\alpha, \beta]$.

From the assumption $[\alpha, \beta] \triangleleft_{[\alpha, \beta]} U$ we get a pair $(r, s) \in R$ satisfying the conditions of the Lemma. Using Lemma 6 we then have $(r, s) \triangleleft_{f} U_{0} \cup \mathcal{C}[\alpha, \beta]$ for some finite subset $U_{0} \subseteq U$. But then, again using the lemma above, $[\alpha, \beta] \triangleleft_{[\alpha, \beta]} U_{0}$.

\footnotetext{
${ }^{3}$ See for example [4, Ch. 3.4], or the cover $\left(I_{n}\right)_{n \in \mathbb{N}}$ defined in the proof of Theorem 9.
} 


\subsection{Heine-Borel and Spatiality}

The relation between the point-free and point-wise Heine-Borel properties has to do with the notion of formal versus point-wise cover. Any formal cover $a \triangleleft \mathcal{X} U$ is a point-wise cover in the sense that $\alpha \in \operatorname{ext}[a]$ implies $\alpha \in \operatorname{ext}[U]$ for any formal point $\alpha$ in $\mathcal{X}$. The converse implication in general requires non-constructive principles. It is well known that for the localic reals this is the case if and only if its formal space is locally compact, i.e. the unit interval [0,1] is compact [9]. For the sake of completeness we give a proof in the framework of formal topology.

For a formal topology $\mathcal{X}=(X, \leqslant, \triangleleft \mathcal{X})$ we define the spatial cover:

$$
a \triangleleft P t(\mathcal{X}) U \stackrel{\text { def }}{\Longleftrightarrow}(\forall \alpha \in \operatorname{Pt}(\mathcal{X}))(a \in \alpha \Longrightarrow \alpha \gamma U) .
$$

As noted above we always have $\triangleleft \mathcal{X} \subseteq \triangleleft_{\mathrm{Pt}(\mathcal{X})}$, but not necessarily the converse.

Definition 15 A formal topology $\mathcal{A}=(A, \leqslant, \triangleleft)$ is said to be spatial if for all $a \in A$, $U \subseteq A$

$$
a \triangleleft \operatorname{Pt}(\mathcal{A}) U \Longrightarrow a \triangleleft_{\mathcal{A}} U \text {. }
$$

This notion of spatiality coincides with the usual one of locale theory. See [10] for a detailed account of spatiality in formal topology. The covering relation for the formal reals has the following connection to its spatial covering relation, which follows from items 1-3 of Lemma 6.

Lemma 16 For all $(p, q) \in R$ and $U \subseteq R$ we have

$$
(p, q) \triangleleft U \Longleftrightarrow(\forall(r, s)<(p, q))\left(\exists U_{0} \in \operatorname{Fin}(U)\right)\left((r, s) \triangleleft \operatorname{Pt}(\mathcal{R}) U_{0}\right)
$$

Proposition 17 Spatiality of $\mathcal{R}$ is equivalent to $\mathbf{H B}$ for $\operatorname{Pt}(\mathcal{R})$.

Proof Suppose $\mathbf{H B}$ holds for $\operatorname{Pt}(\mathcal{R})$ and suppose $(p, q) \triangleleft \operatorname{Pt}(R) U$, so that $\operatorname{ext}[(p, q)] \subseteq$ $\operatorname{ext}[U]$. If $(r, s)<(p, q)$, then $[r, s] \subseteq \operatorname{ext}[U]$ so there is a finite subset $U_{0} \subseteq U$ such that $[r, s] \subseteq \operatorname{ext}\left[U_{0}\right]$ and hence $\operatorname{ext}[(r, s)] \subseteq[r, s] \subseteq \operatorname{ext}\left[U_{0}\right]$, i.e. $(r, s) \triangleleft \operatorname{Pt}(\mathcal{R}) U$. But then $(p, q) \triangleleft U$ by Lemma 16 .

Conversely, if $\mathcal{R}$ is spatial, and $[0,1] \subseteq \operatorname{ext}[U]$, then there is $(p, q) \in R$ with $[0,1] \subseteq \operatorname{ext}[(p, q)] \subseteq \operatorname{ext}[U]$, i.e. $(0,1)<(p, q)$ (ext[U] being open). So $(p, q) \triangleleft \operatorname{Pt}(\mathcal{R}) U$ which implies $(p, q) \triangleleft U$. If we let $(r, s)<(p, q)$ with $(0,1)<(r, s)$ we then have by Lemma 16 that $(r, s) \triangleleft \operatorname{Pt}(\mathcal{R}) U_{0}$ for some finite subset $U_{0} \subseteq U$. That is $[0,1] \subseteq \operatorname{ext}[(r, s)] \subseteq \operatorname{ext}\left[U_{0}\right]$ 
Remark 18 By $\mathbf{H B}$ for $\operatorname{Pt}(\mathcal{R})$ we here mean the Heine-Borel principle restricted to covers $\left(I_{j}\right)_{j \in J}$ consisting of intervals with rational endpoints. Such a covering is precisely ext $[U]$ for some $U \subseteq R$.

\subsection{Vitali's Covering Theorem}

Recall that a (point-wise) Vitali cover of an interval $J \subseteq \mathbb{R}$ is a family of intervals $\mathcal{V}$ such that for any $x \in J$ and $k>0$, there exists $I \in \mathcal{V}$ with $x \in I$ and $|I|<k$. The analogue property for covers in the formal reals $\mathcal{R}=(R, \leqslant, \triangleleft)$ is given by the following definition.

Definition 19 Let $V \subseteq R$ and $(p, q) \in R$. If for every $(r, s) \leqslant(p, q)$ we have

$$
(r, s) \triangleleft V \cap \downarrow\{(r, s)\}
$$

we say that $V$ is a formal Vitali cover of $(p, q)$. We say that $V$ is a Vitali cover of $U \subseteq R$ if $V$ is a Vitali cover of every $(p, q) \in U$.

Any formal Vitali cover $V$ of $(p, q)$ is clearly also a formal cover $(p, q) \triangleleft V$. Moreover, every formal Vitali cover is always a point-wise Vitali cover.

Lemma 20 If $(p, q) \triangleleft V$ is a formal Vitali cover then $(p, q) \triangleleft \operatorname{Pt}(\mathcal{R}) V$ is a Vitali cover.

Proof Suppose $(p, q) \triangleleft V$ is a formal Vitali cover and let $\alpha \in \operatorname{ext}[(p, q)]$ and $k>0$. Then $(p, q) \in \alpha$, and by Lemma 8 we can find $(r, s) \in \alpha \cap \downarrow\{(p, q)\}$ with $s-r<k$. By (4) we have $(r, s) \triangleleft V \cap \downarrow\{(r, s)\}$ and since $(r, s) \in \alpha$ there is $(u, v) \in V \cap \downarrow\{(r, s)\}$ such that $(u, v) \in \alpha$ (using (P3)), moreover $v-u \leq s-r<k$.

Assuming spatiality of $\mathcal{R}$ the converse also holds.

Lemma 21 Suppose $\mathcal{R}$ is spatial and that $(p, q) \triangleleft \operatorname{Pt}(\mathcal{R}) V$ is a Vitali cover. Then $(p, q) \triangleleft V$ is a formal Vitali cover.

Proof Let $V \subseteq R$ be a point-wise Vitali cover of $(p, q) \in R$, i.e. $\operatorname{ext}[(p, q)]$ is Vitali covered by the collection of intervals $\operatorname{ext}[(u, v)]$ where $(u, v) \in V$. If $(r, s) \leqslant(p, q)$ and $\alpha \in \operatorname{ext}[(r, s)]$ then with $k \leq \min (\alpha-r, s-\alpha)$ there is $(u, v) \in V$ with $\alpha \in \operatorname{ext}[(u, v)]$ and $v-u<k$. Then $(u, v) \leqslant(r, s)$, and hence $(u, v) \in V \cap \downarrow\{(r, s)\}$ which implies $(r, s) \triangleleft \operatorname{Pt}(\mathcal{R}) V \cap \downarrow\{(r, s)\}$. But then $(r, s) \triangleleft V \cap \downarrow\{(r, s)\}$ by spatiality. 
We also note that assuming spatiality only for Vitali covers in fact implies spatiality for all covers.

Lemma 22 If for all $(p, q) \in R$ and $V \subseteq R$

$$
(p, q) \triangleleft_{\mathrm{Pt}(\mathcal{R})} V \text { Vitali cover } \Longrightarrow(p, q) \triangleleft V,
$$

then $\mathcal{R}$ is spatial.

Proof Suppose $(p, q) \triangleleft \operatorname{Pt}(\mathcal{R}) V$ and let $\alpha \in \operatorname{ext}[(p, q)]$. Then there is $(u, v) \in V$ such that $\alpha \in \operatorname{ext}[(u, v)]$. Since there are arbitrarily small $(r, s) \in \alpha \cap \downarrow\{(u, v)\}$ (using Lemma 8) we see that ext $[\downarrow V]$ is a point-wise Vitali cover of ext $[(p, q)]$. By (5) we then have $(p, q) \triangleleft \downarrow V$ from which $(p, q) \triangleleft V$ follows by (Tra), since $\downarrow V \triangleleft V$.

By a formal Vitali cover $V$ of an interval $[\alpha, \beta]$ (considered as a closed subspace of $\mathcal{R}$ ) we mean a subset $V \subseteq R$ such that

$$
R \triangleleft_{[\alpha, \beta]} V
$$

is a formal Vitali cover, i.e. for every $(p, q) \in R$ we have $(p, q) \triangleleft_{[\alpha, \beta]} V \cap \downarrow\{(p, q)\}$.

Lemma 23 Suppose $V$ is a Vitali cover of $[\alpha, \beta]$ and that

$$
r<\alpha<p<s<u<q<\beta<v,
$$

for $r, p, s, u, q, v \in \mathbb{Q}$. Then $[\alpha, \beta] \triangleleft_{[\alpha, \beta]}\{(r, s),(u, v)\} \cup(V \cap \downarrow\{(p, q)\})$.

Proof If $(a, b) \in R$, then $(a, b) \triangleleft_{[\alpha, \beta]}\{(r, s),(p, q),(u, v)\}$, moreover $(p, q) \triangleleft_{[\alpha, \beta]} V \cap$ $\downarrow\{(p, q)\}$ by the Vitali property, and by (Tra) we then have

$$
(a, b) \triangleleft_{[\alpha, \beta]}\{(r, s),(u, v)\} \cup(V \cap \downarrow\{(p, q)\}) .
$$

Lemma 24 Suppose $V$ is a Vitali cover of $[\alpha, \beta]$, for some $\alpha<\beta$ in $\operatorname{Pt}(\mathcal{R})$, then there exists a finite subset $V_{0}=\left\{\left(u_{1}, v_{1}\right), \ldots,\left(u_{n}, v_{n}\right)\right\} \subseteq V$ of pairwise disjoint elements such that

$$
\mu\left(\operatorname{ext}\left[V_{0}\right]\right)>\frac{1}{4}(\beta-\alpha),
$$

$\alpha<u_{i}<v_{i}<\beta$ for all $1 \leq i \leq n$. 
Proof Let $r<\alpha<s<u<\beta<v$ such that both

$$
s-\alpha<\frac{1}{8}(\beta-\alpha) \text { and } \beta-u<\frac{1}{8}(\beta-\alpha)
$$

Then, using Lemma 23,

$$
[\alpha, \beta] \triangleleft_{[\alpha, \beta]}\{(r, s),(u, v)\} \cup(V \cap \downarrow\{(p, q)\})
$$

for any $(p, q)$ with $\alpha<p<s$ and $u<q<\beta$. By Theorem 13 (Heine-Borel for $\mathcal{R}$ ) we then have a finite subset $V_{0}^{\prime} \subseteq V \cap \downarrow\{(p, q)\}$ such that

$$
[\alpha, \beta] \triangleleft_{[\alpha, \beta]}\{(r, s),(u, v)\} \cup V_{0}^{\prime} .
$$

Say we have $\left.V_{0}^{\prime}=\left\{\left(u_{1}, v_{1}\right), \ldots,\left(u_{n}, v_{n}\right)\right)\right\}$, then $\left(u_{i}, v_{i}\right) \leqslant(p, q)$ for each $1 \leq i \leq n$, and $(s, u) \triangleleft_{[\alpha, \beta]} V_{0}^{\prime}$ (by (Loc) and (Tra)) which implies $(s, u) \triangleleft V_{0}^{\prime}$ since $\alpha<s<u<\beta$. By Lemma 6 we then have

$$
\mu\left(\operatorname{ext}\left[V_{0}^{\prime}\right]\right)>(\beta-\alpha)-\frac{1}{4}(\beta-\alpha)=\frac{3}{4}(\beta-\alpha) .
$$

Now, using lemma 3 we find $V_{0} \subseteq V_{0}^{\prime}$ consisting of pairwise disjoint elements such that

$$
\frac{1}{3} \mu\left(\operatorname{ext}\left[V_{0}^{\prime}\right]\right) \leq \mu\left(\operatorname{ext}\left[V_{0}\right]\right)
$$

As noted in Remark 4 we get the constant $1 / 3$ instead of $1 / 4$ since the intervals here have rational endpoints. But then we have $\mu\left(\operatorname{ext}\left[V_{0}\right]\right)>\frac{1}{4}(\beta-\alpha)$.

Note that the finite set $V_{0}$ in the Lemma only contains intervals $(u, v)$ with ext $[(u, v)] \subseteq$ $[\alpha, \beta]$.

Thus we see that any formal Vitali cover has the property $V_{\frac{3}{4}}$ and then, as explained in the preliminaries, the main theorem follows quite easily.

Theorem 25 (Formal Vitali Covering Theorem) Suppose $V$ is a formal Vitali cover of $[\alpha, \beta]$, for some $\alpha<\beta$ in $\operatorname{Pt}(R)$. Then there is a sequence $\left\{\left(u_{i}, v_{i}\right): i \geq 1\right\}$ of pairwise disjoint elements of $V$ with $\alpha<u_{i}<v_{i}<\beta$ for all $1 \leq i \leq n$ and for each $\varepsilon>0$ there is $N \in \mathbb{N}$ such that

$$
\sum_{i=1}^{N} v_{i}-u_{i}>\beta-\alpha-\varepsilon .
$$




\section{Discussion}

We would like to conclude the paper by briefly sketching the outlines of yet another possible, constructive, view of VCT.

Recursive mathematics is roughly divided into two schools that are known as the Russian one, mentioned above, and the Polish one, which also includes Weihrauch's popular Type Two Effectivity approach [19]. Both schools are mainly interested in computable (recursive) objects. The difference lies in whether or not one assumes a classical or a constructive meta-theory, and hence whether or not non-computable objects are "ignored" or not. For a practitioner of RUSS all functions between natural numbers are computable - a non-computable function between natural numbers simply cannot be defined. Along the same lines in RUSS all real numbers are computable. ${ }^{4}$ This view leads to the existence of strange objects, such as a bounded, increasing sequence of real numbers that does not converge to any real number [17]. Of course, if one assumes the Polish standpoint one would argue that this sequence converges to a non-computable real number, which in RUSS does not exist. Similarly, the key to the counterexample in Section 3 relies on the fact, that from the Polish point of view, the Vitali cover only covers the computable real numbers and not all real numbers. It would be more in the tradition of the Polish school to assume that a Vitali cover is a cover for all real numbers. Since the classical proof of the theorem guarantees the existence of a finite, disjoint, sufficiently large subcover of such a Vitali cover, and, furthermore one can effectively enumerate all such subcovers, one can compute VCT by a simple unbounded search that is guaranteed to terminate.

\section{Acknowledgements}

This work was stimulated by the report "towards a constructive proof of the Vitali Covering Theorem", written by M. Jordens under the supervision of D.S. Bridges, as part of a Summer research project at the University of Canterbury, New Zealand. The research was initiated during an exchange funded through the EU Marie Curie IRSES project 'CONSTRUMATH'. The second author was supported by FMB, the Swedish Graduate School in Mathematics and Computing.

The authors would like to thank the anonymous referees for helpful comments that helped improve the content and style of the paper.

\footnotetext{
${ }^{4}$ i.e. computable Cauchy sequences of rational numbers with a computable modulus.
} 


\section{References}

[1] P Aczel, M Rathjen, Notes on constructive set theory, Technical Report 40, Institut Mittag-Leffler, Royal Swedish Academy of Sciences, 2001; available at http://www.ml.kva.se/preprints/archive/2000-2001/2000-2001-40.pdf.

[2] M J Beeson, Foundations of Constructive Mathematics, Ergebnisse der Mathematik und ihrer Grenzgebiete, Springer Verlag, 1985.

[3] E Bishop, D S Bridges, Constructive Analysis, Grundlehren der matematischen Wissenschaften 279, Springer-Verlag, Berlin, 1985.

[4] DS Bridges, F Richman, Varieties of Constructive Mathematics, London Mathematical Society Lecture Note Series 97, Cambridge University Press,1987; doi: $10.2277 / 0521318025$.

[5] D K Brown, M Giusto, S G Simpson, Vitali's theorem and WWKL, Arch. Math. Logic 41 (2002), 191-206; doi:10.1007/s001530100100.

[6] J C Burkill, The Lebesgue Integral, Cambridge Tracts in Mathematics and Mathematical Physics 40, Cambridge University Press, 1953; doi: 10.1017/CBO9780511566127.

[7] J Cederquist, S Negri, A constructive proof of the Heine-Borel covering theorem for formal reals, in: Types for proofs and programs (Torino, 1995), (eds. S Berardi, M Coppo), Lecture Notes in Computer Science 1158, Springer, Berlin, 1996, 62-75; doi:10.1007/3-540-61780-9_62.

[8] H Diener, Compactness under constructive scrutiny, $\mathrm{PhD}$ thesis, University of Canterbury, Christchurch, New Zealand, 2008; available at http://hdl. handle.net/10092/1823.

[9] MP Fourman, R Grayson, Formal spaces, in: L.E.J. Brouwer Centenary Symposium. Proceedings of the Conference held in Noordwijkerhout, 8-13 June, 1981, (eds. D van Dalen, A Troelstra), Studies in Logic and the Foundations of Mathematics 110, North-Holland, 1982, 107-122; doi:10.1016/S0049-237X(09)70126-0.

[10] N Gambino, P Schuster, Spatiality for formal topologies, Mathematical Structures in Comp. Sci. 17 (2007), 65-80; doi:10.1017/S0960129506005810.

[11] P T Johnstone, Stone Spaces, Cambridge Studies in Advanced Mathematics 3, Cambridge University Press, Cambridge, 1982; doi:10.2277/0521337798.

[12] H Lebesgue, Leçons sur l'Intégration et la Recherche des Fonctions Primitives, GauthierVillars, Paris, 1904.

[13] S Negri, D Soravia, The continuum as a formal space, Archive for Mathematical Logic 38 (1999), 423-447; doi:10.1007/s001530050149.

[14] T Nemoto, Weak weak König's lemma in constructive reverse mathematics, in: Proceedings of the 10th Asian Logic Conference, Kobe University in Kobe, Hyogo, Japan, 1-6 September, 2008, World Scientific, 2009, 263-270; doi:10.1142/9789814293020_0010. 
[15] G Sambin, Intuitionistic formal spaces - a first communication, in: Mathematical Logic and its Applications (Druzhba, 1986), (ed. D Skordev), Plenum, New York, 1987, 187-204.

[16] S G Simpson, Subsystems of Second Order Arithmetic (2nd Edition), Cambridge University Press, 2009; doi:10.1017/CBO9780511581007.

[17] E Specker, Nicht konstruktiv beweisbare Sätze der Analysis, J. Symbolic Logic 14 (1949), 145-158.

[18] G Vitali, Sui gruppi di punti e sulle funzioni di variabili reali, Atti Accad. Sci. Torino 43 (1908), 229-246.

[19] K Weihrauch, Computable Analysis : An Introduction, Texts in Theoretical Computer Science, Springer, 2000.

[20] X Yu, S G Simpson, Measure theory and weak König's lemma, Arch. Math. Logic 30 (1990), 171-180; doi:10.1007/BF01621469.

Fakultät IV: Mathematik, Universität Siegen

Emmy-Noether-Campus, Walter-Flex-Str. 3, 57068 Siegen, Germany

Department of Mathematics, Uppsala University

P.O. Box 480, SE-751 06 Uppsala, Sweden

diener@math.uni-siegen.de, anton.hedin@math.uu.se

Received: 20 December $2010 \quad$ Revised: 28 November 2011 